\title{
Técnica de Takeuchi en el origen anómalo de la arteria coronaria izquierda de la arteria pulmonar
}

\author{
Takeuchi technique in the anomalous origin of the left coronary artery from the pulmonary \\ artery
}

\author{
Carlos Domínguez-Massa*, Tomás Heredia-Cambra, Alberto Berbel-Bonillo, Óscar R. Blanco-Herrera, \\ José A. Montero-Argudo y Fernando Hornero-Sos \\ Servicio de Cirugía Cardiovascular, Hospital Universitario y Politécnico La Fe. Valencia, España
}

\section{Introducción}

Las anomalías de las arterias coronarias representan el $2.2 \%$ de los defectos cardíacos congénitos ${ }^{1}$, siendo la más frecuente el origen anómalo de la arteria coronaria izquierda de la arteria pulmonar (conocida por su acrónimo en inglés ALCAPA: anomalous origin of the left coronary artery from the pulmonary artery) $)^{1}$. EI ALCAPA o síndrome de Bland-White-Garland tiene una incidencia de 1 de cada 30,000 a 300,000 recién nacidos $^{1-3}$, con una mortalidad del $90 \%$ en el primer año de vida ${ }^{2}$. En el adulto, suelen estar asintomáticos o presentar insuficiencia mitral, cardiopatía isquémica, insuficiencia cardíaca o arritmias ventriculares malignas (causa de muerte súbita en el $80-90 \%)^{2}$. La mayoría de los pacientes adultos presenta dilatación significativa de la arteria coronaria derecha debida al aumento del flujo a la arteria coronaria izquierda asociado con extensas colaterales intercoronarias. Sin embargo, una arteria coronaria derecha gigante asociada al síndrome de ALCAPA es inusual ${ }^{3}$. El mecanismo de la insuficiencia mitral en el ALCAPA consiste en la disfunción de los músculos papilares por isquemia (habitualmente, el músculo papilar posterior), pero otras veces es consecuencia de la dilatación del anillo mitral secundariamente a la configuración esferoidal que adopta el ventrículo izquierdo isquémico, lo que causa un defecto de coaptación de los velos mitrales'.

El diagnóstico se realiza con angiografía coronaria, pero la tomografía computarizada (TC)-coronariografía también establece un diagnóstico claro dando además información anatómica adicional antes de la cirugía 4 . La cirugía está recomendada en todos los pacientes, incluso si están asintomáticos ${ }^{1}$.

\section{Descripción del caso}

Mujer de 44 años, que realizando ejercicio físico intenso experimentó una pérdida súbita de la conciencia, iniciándose maniobras de reanimación cardiopulmonar básica hasta la llegada del Servicio de Asistencia Médica Urgente. Se documentó fibrilación ventricular que fue revertida con desfibrilación eléctrica. La paciente fue trasladada al Hospital. El electrocardiograma (ECG) estaba en ritmo sinusal, a unos $70 \mathrm{lpm}$, con eje-30 y signos de hipertrofia ventricular izquierda. La ecocardiografía mostró insuficiencia mitral ligera-moderada sin otras alteraciones valvulares. El ventrículo izquierdo estaba ligeramente dilatado con hipocinesia de los segmentos medio y basal de la cara anterior y de la zona basal del septo, preservando la función sistólica. Se realizó coronariografía urgente que reveló ectasia

\section{Correspondencia:}

${ }^{*}$ Carlos Domínguez-Massa

Av. de Fernando Abril Martorell 106, 


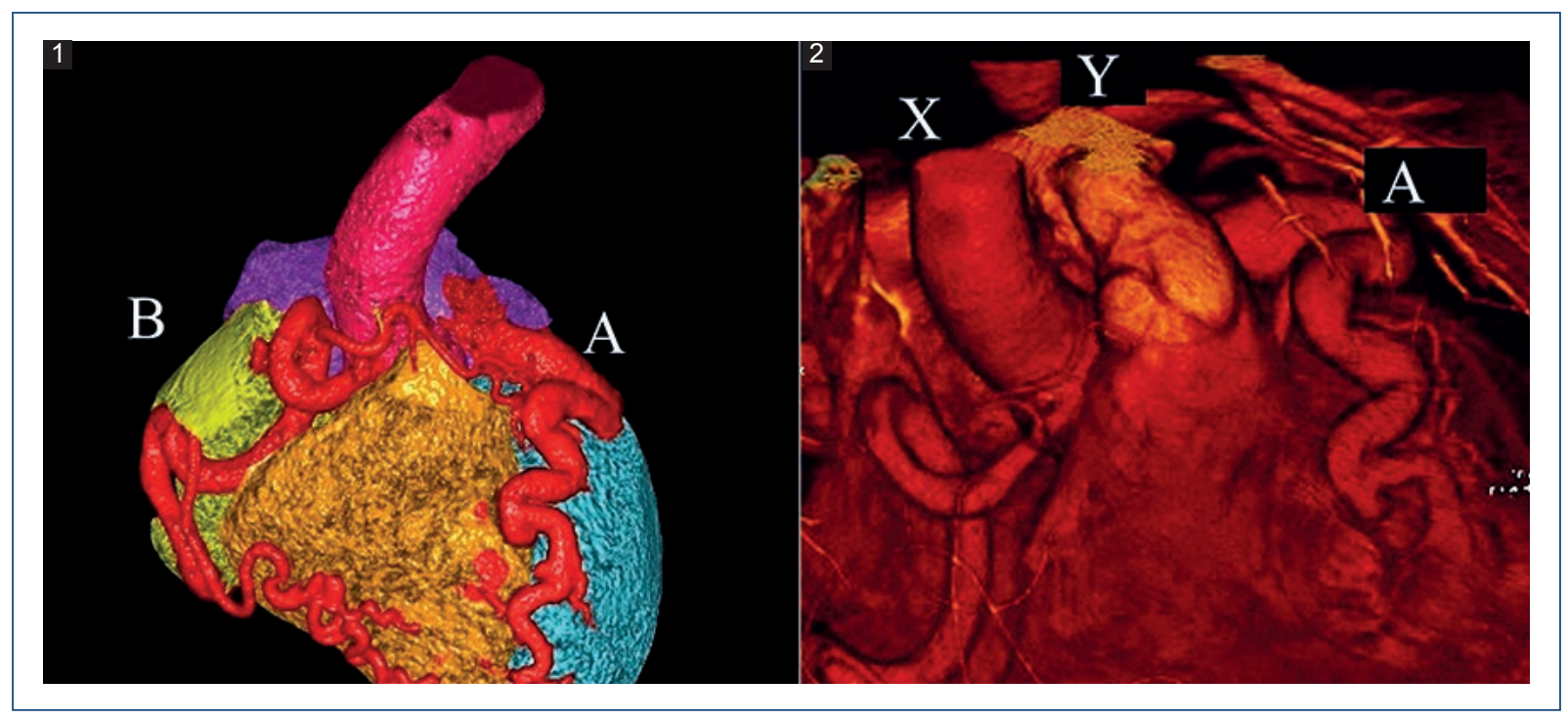

Figura 1. Reconstrucción de la TC-coronariografía. 1: Preoperatorio. La arteria coronaria izquierda (A) se origina en la arteria pulmonar. La arteria coronaria derecha (B) es ectásica con colaterales a la arteria coronaria izquierda. 2: Postoperatorio. La arteria coronaria izquierda está tunelizada a través de la arteria pulmonar (Y) a la aorta (X).

severa de la arteria coronaria derecha con extensas colaterales a la arteria coronaria izquierda.

Estabilizada la paciente, se realizó una TC-coronariografía (Fig. 1.1), que fue diagnóstica del síndrome de ALCAPA: la arteria coronaria izquierda nacía a nivel lateral izquierdo de la arteria pulmonar y se rellenaba a través de numerosas colaterales dependientes de ramas de la arteria coronaria derecha cruzando el miocardio ventricular. Además, había importante dilatación y tortuosidad de todas las arterias coronarias, en relación con el desarrollo de colaterales en el contexto de isquemia crónica. Como estudio complementario, se realizó una resonancia magnética que demostró dilatación ventricular con infarto subendocárdico de las caras anterior, anteroseptal y anterolateral (todos segmentos viables), asociado con insuficiencia mitral moderada debido a hipocinesia del músculo papilar anterolateral en relación a isquemia crónica.

Se programó para cirugía preferente, realizándose la técnica de Takeuchi a través de esternotomía media completa. Tras establecer el bypass cardiopulmonar, se realizó arteriotomía transversa de la arteria pulmonar visualizándose la válvula pulmonar y el origen anómalo de la arteria coronaria izquierda en el tronco pulmonar, apreciándose el nacimiento de la arteria coronaria izquierda a nivel lateral izquierdo de la arteria pulmonar. Se obtuvo una lengüeta de arteria pulmonar a nivel del origen anómalo de la arteria coronaria izquierda
(Fig. 2A). Con un punch, se realizó un agujero en la aorta y en la arteria pulmonar para la realización de la ventana aortopulmonar (Fig. 2B). Con la lengüeta de arteria pulmonar se procedió a tunelizar un conducto desde el origen de la arteria coronaria izquierda hasta la ventana aortopulmonar (Fig. 2C). Se utilizó cardioplejía anterógrada para comprobar la estanqueidad de la sutura realizada. Por último, se cerró la arteria pulmonar utilizando un injerto de pericardio autólogo para evitar su estenosis (Fig. 2D).

La paciente fue extubada a las 6 horas del procedimiento. Presentó como única complicación postoperatoria dos episodios de fibrilación auricular con regular tolerancia hemodinámica. El primer episodio revirtió a ritmo sinusal con cardioversión farmacológica y el segundo con cardioversión eléctrica. En las primeras semanas se realizó una TC-coronariografía (Fig. 1.2), que confirmaba la permeabilidad de la arteria coronaria izquierda tunelizada a través de la arteria pulmonar. En la ecocardiografía, el ventrículo izquierdo permanecía dilatado y persistía una insuficiencia mitral ligera. En la revisión de la paciente a los 6 meses postoperatorios se encontró una buena evolución clínica, tolerando progresivamente el esfuerzo físico.

\section{Discusión}

La cirugía está recomendada en todos los pacientes con ALCAPA, incluso si están asintomáticos. Hay varias 


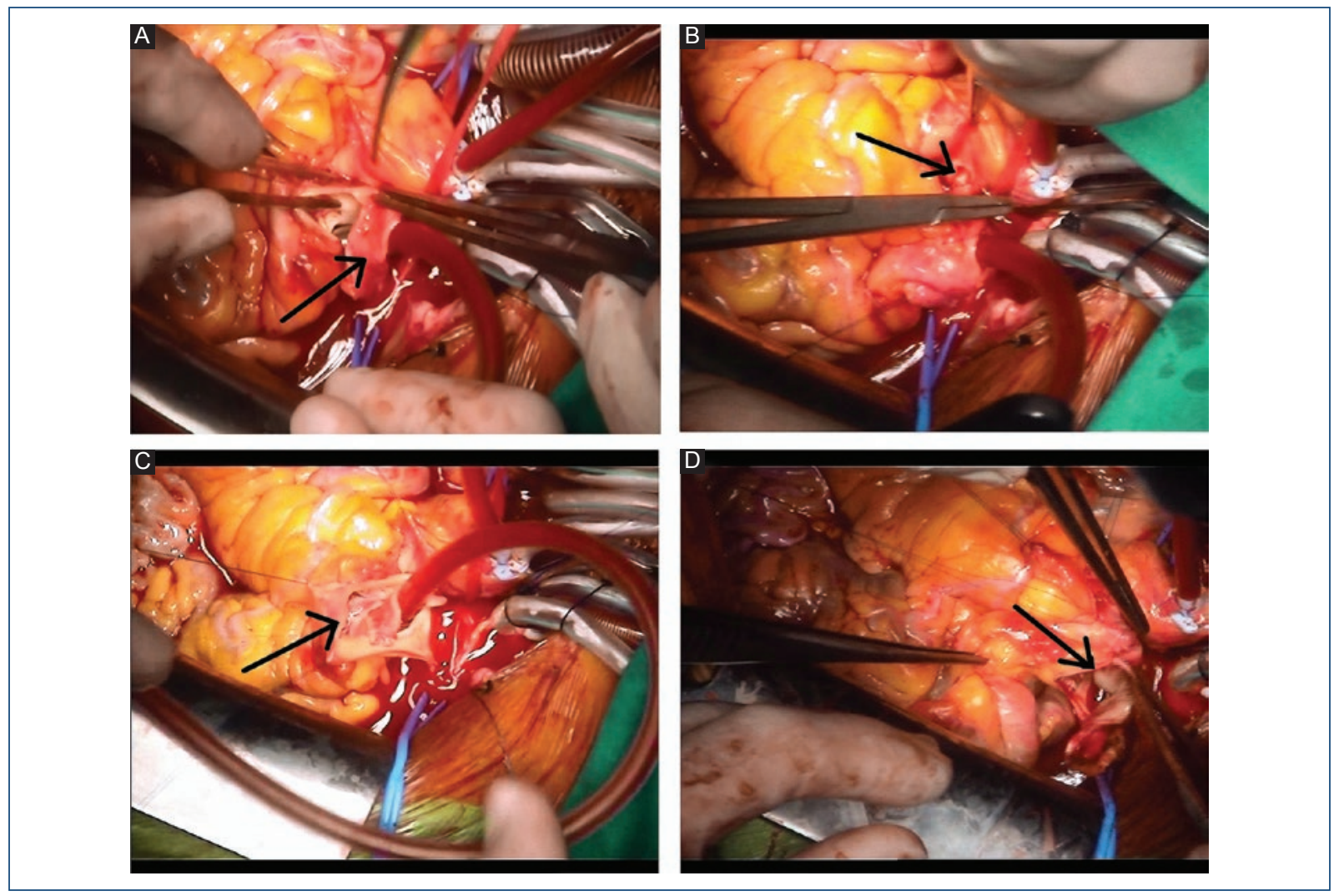

Figura 2. Fotografías del campo quirúrgico. Se marca con una flecha el paso quirúrgico explicado. A: Arteriotomía pulmonar para visualizar la válvula pulmonar y el origen de la arteria coronaria izquierda. B: Realización de la ventana aortopulmonar. C: Tunelización de la arteria coronaria izquierda a través de la arteria pulmonar, con la lengüeta de arteria pulmonar, para que se origine en la aorta por la ventana aortopulmonar realizada. D: Cierre y ampliación de la arteria pulmonar con parche autólogo de pericardio.

opciones quirúrgicas, que se dividen según se deje un solo sistema coronario o si se restablecen los dos sistemas coronarios, con mejores resultados a largo pla$\mathrm{zo}^{1,4}$. Las estrategias más usadas son el reimplante directo y la técnica de Takeuchi5. El uso de la arteria pulmonar en la técnica de Takeuchi parece mejor que el uso de pericardio u otro material sintético debido a que el pericardio conlleva mayor riesgo de formación de aneurismas debido a alta presión, y los materiales sintéticos son más trombogénicos ${ }^{6}$.

El bypass de la arteria coronaria izquierda con oclusión o ligado del origen anómalo es una estrategia alternativa. No obstante, hay dudas sobre el flujo competitivo de las colaterales que pueden afectar a la permeabilidad del injerto, teniendo además el inconveniente de la oclusión del injerto a medio-largo plazo. Por ello, la cirugía de revascularización coronaria con el cierre de la arteria coronaria anómala debe ser reservada para aquellos casos en los que la transferencia coronaria no es factible ${ }^{7}$.
La cirugía de la válvula mitral en los pacientes con ALCAPA sigue siendo controvertida. Algunos autores no recomiendan la cirugía valvular mitral durante el reimplante coronario, indicando que la insuficiencia mitral mejora o desaparece completamente posteriormente, particularmente en la infancia. Por el contrario, otros autores recomiendan realizar una anuloplastia mitral simultáneamente cuando existe insuficiencia mitral moderada a severa. La decisión de corregir la insuficiencia mitral debe ser flexible y basada en sus causas subyacentes, pues la insuficiencia mitral residual se ha identificado como un factor de riesgo de reintervención, pero no de muerte ${ }^{8,9}$.

\section{Conclusión}

La cirugía del ALCAPA debe individualizarse en relación con la edad, pronóstico y anatomía de cada paciente. La técnica de Takeuchi se elige cuando no es posible el reimplante coronario por una anatomía 
desfavorable, como el caso presentado, que tenía un nacimiento a nivel lateral izquierdo de la arteria pulmonar. No hay evidencia en la literatura de la actitud correcta sobre la insuficiencia mitral, que suele resolverse al cesar el evento isquémico desencadenante.

\section{Financiamiento}

La presente investigación no ha recibido ayudas específicas provenientes de agencias del sector público, sector comercial o entidades sin ánimo de lucro.

\section{Conflicto de intereses}

Los autores declaran no tener conflicto de intereses.

\section{Responsabilidades éticas}

Protección de personas y animales. Los autores declaran que para esta investigación no se han realizado experimentos en seres humanos ni en animales.

Confidencialidad de los datos. Los autores declaran que han seguido los protocolos de su centro de trabajo sobre la publicación de datos de pacientes.
Derecho a la privacidad y consentimiento informado. Los autores declaran que en este artículo no aparecen datos de pacientes.

\section{Bibliografía}

1. Ramírez S, Curi-Curi PJ, Calderón-Colmenero J, García J, Britton C Erdmenger J, et al. Resultados del reimplante coronario para la corrección del origen anómalo de la coronaria izquierda a partir de la arteria pulmonar. Rev Esp Cardiol. 2011;64(8):681-7.

2. Kothari J, Lakhia K, Solanki P, Parmar D, Boraniya H, Patel S. Anomalous origin of the left coronary artery from the pulmonary artery in adulthood: Challenges and outcomes. Korean J Thorac Cardiovasc Surg. 2016;49:383-6.

3. Bajona P, Maselli D, Dore R, Minzioni G. Anomalous origin of the left main artery from the pulmonary artery: Adult presentation with systemic collateral supply and giant right coronary artery aneurysm. J Thorac Cardiovasc Surg. 2007;134:518-20.

4. Joshi SV, Naik AV, Bhalgat PS, Chavan B, Salvi P. An experience with off pump technique for repair of anomalous left coronary artery from pulmonary artery (ALCAPA). Indian Heart J. 2016;68:704-8.

5. Isomatsu Y, Imai Y, Shin'oka T, Aoki M, Iwata Y. Surgical intervention for anomalous origin of the left coronary artery from the pulmonary artery: The Tokyo Experience. J Thorac Cardiovasc Surg. 2001;121:792-7.

6. Kattach H, Anastasiadis K, Jin XY, Pillai R. Two-conduit repair for anomalous origin of the left coronary artery from the pulmonary artery in an adult. J Thorac Cardiovasc Surg. 2004;128:641-2.

7. Rajbanshi BG, Burkhart HM, Schaff HV, Daly RC, Phillips SD, Dearani JA Surgical strategies for anomalous origin of coronary artery from pulmonary artery in adults. J Thorac Cardiovasc Surg. 2014;148:220-4.

8. Alexi-Meskishivili V, Nasseri BA, Nordmeyer S, Schmitt B, Weng YG Böttcher W, et al. Repair of anomalous origin of the left coronary artery from the pulmonary artery in infants and children. $J$ Thorac Cardiovasc Surg. 2011;142:868-74.

9. Pérez-Negueruela C, Arango-Posada C, César S, Bartrons J, Carretero J, Sarquella-Brugada G, et al. Nueve casos de origen anómalo de una arteria coronaria. Cir Cardiov. 2014;21:204-8. 